\title{
0 domínio de organização do conhecimento na base BRAPCI: uma análise estatística
}

\author{
Miguel Romeu Amorim Neto * \\ Marcia Heloisa Tavares de Figueredo Lima **
}

Artículo recibido:

17 de junio de 2014.

Artículo aceptado:

24 de febrero de 2015.

\section{Resumo}

Investigação analítico-quali/quantitativa detectando o domínio de Organização do Conhecimento frente à produção brasileira de artigos de periódicos de Ciência da Informação a partir de levantamentos realizados na Base de Dados Referencial de Artigos de Periódico em Ciência da Informação. O objetivo geral da pesquisa foi analisar comparativamente a ocorrência numérica absoluta e relativa de artigos de periódicos brasileiros da área de CI sobre a temática Organização do Conhecimento cobertos na base BRAPCI de 1972 a 2012 através de recortes temático-conceituais. Apresenta conceituações para a compreensão da Organização do Conhecimento, a partir da abordagem da Aná-

* Universidade Federal do Rio de Janeiro, Rio de Janeiro RJ, Brasil. miguel@sibi.ufrj.b

** Universidade Federal Fluminense, Niterói RJ, Brasil. marciahelolima@gmail.com

INVESTIGACIÓN BIBLIOTECOLÓGICA, Vol.30, Núm.70, septiembre/diciembre, 2016, México, ISSN: 0187-358X. pp. 83-104 
lise de Domínio de Hjørland e Albrechtsen (1995), a qual permitiu que fossem identificados os conceitos pertencentes da OC. Para atender aos objetivos de pesquisa, utilizaram-se técnicas quantitativas de avaliação da produção científica como método de análise dos dados recuperados na BRAPCI e análise qualitativa dos resultados. Estas sinalizaram que o Domínio de OC está em expansão no Brasil ao contrário do cenário internacional.

Palavras-chave: Organização do Conhecimento; Base de dados; BRAPCI; Comunicação científica; Bibliometria; Análise quantitativa; Produtividade Relativa; Meta-análise.

\section{Abstract}

Domain of knowledge organization represented in the BRAPCI base: a statistical analysis

Miguel-Romeu Amorim-Neto and Marcia-Heloisa Tavares-de-Figueredo-Lima

Investigation qualitative and quantitative analysis detecting the domain of Knowledge Organization front of the whole area of information science from surveys conducted in Referential Database Journal Articles in Information Science. The objective of the research is to analyze the occurrence numerical absolute and relative articles of Brazilian journals of information science on the topic covered in the Knowledge Organization based BRAPCI 1972-2012 through conceptual-themed cutouts. Presents concepts for understanding the Organization of Knowledge, from the approach of Domain Analysis of Hjørland and Albrechtsen (1995), which allowed them to be identified concepts of belonging OC. To meet the research objectives, we used quantitative techniques for assessing the scientific literature as a method of analyzing the data retrieved in BRAPCI and qualitative analysis. This signaled that the domain OC is expanding in Brazil unlike the international scene.

Keywords: Knowledge Organization; Database; BRAPCI; Scientific Communication; Bibliometrics; Quantitative analysis; Relative Productivity; Metaanalysis. 


\section{INTRODUÇÃO}

— ste trabalho relata resultados de pesquisa que teve como meta verificar a _importância relativa da Organização do Conhecimento nos periódicos de Ciência da Informação no Brasil, considerando que diversas pesquisas sinalizavam uma diminuição da importância relativa do domínio de OC em relação a outras temáticas.

Da inicial (e quase exclusiva) preocupação sobre as formas de tratar a informação, o que chamamos de domínio de Tratamento da Informação (TI), a Ciência da Informação (CI), a partir de 1985 vê um movimento de reflexão epistemológica de autores na CI que mapearam os temas de pesquisa privilegiados no campo, demonstrando, a partir da identificação das temáticas centrais de artigos de periódicos, que mudam os focos da pesquisa na área e há um crescente interesse por outras temáticas.

Nos parágrafos seguintes apresentamos um resumo de seis artigos que apresentam propostas de esforços classificatórios com sentido epistemológico e nos demonstram essa mudança.

Atkins (1988) em um estudo com um recorte temporal de 10 anos - 1975 1984 - nas revistas consideradas mais prestigiadas pela comunidade (College CL Research Libraries, Library Quarterly, Journal of Academic Librarianship, Information Technology and Libraries, Library Resources CL Technical Services, Library Trends, ASIS Journal, Library Journal e American Libraries), coletou dados de 2705 artigos, chegando a um total de 4688 assuntos reduzidos ao final da pesquisa em uma lista de 57 assuntos (list of subjects) dominantes. Suas conclusões foram que o assunto mais frequente é a gestão de bibliotecas, seguido por uma grande concentração de assuntos relacionados com a automação de acervos, como recuperação da informação, bancos de dados, catalogação, automação de bibliotecas, tecnologia e métodos de investigação. Devemos ressaltar que o único ponto negativo de seu artigo é que o autor não disponibiliza a categorização dos assuntos de sua lista, e que por isso não pode ser melhor explorado para comparação com outros autores.

Jarvelin e Vakkari (1993), realizando uma pesquisa internacional com quarenta periódicos, distribuída por tópicos, sobre quais as abordagens e métodos têm sido utilizados na Biblioteconomia e Ciência da Informação (BCI ou, em inglês, Libray and Information Science - LIS) a partir de amostras publicadas em 1965, 1975 e 1985, destacam que classificação e indexação, recuperação da informação e as profissões foram naqueles períodos, respectivamente, os tópicos mais publicados. Por outro lado, o estudo demonstrou que houve alterações notáveis nas tendências dos estudos em subcategorias destes tópicos, destacando-se um decréscimo significativo de 
interesse pelos estudos sobre metodologia e análise da BCI (LIS), bem como o decréscimo de interesse sobre a temáticas como armazenamento e recuperação de informação (a publicação sobre a temática "classificação e indexação" passou de $22 \%$ para $6 \%$ ) enquanto houve um aumento de produção de artigos sobre recuperação da informação a partir dos problemas dos usuários (de $4 \%$ para $13 \%$ ). Este estudo nos forneceu um primeiro panorama da BCI como um todo e as subáreas.

No estudo desses autores foi possível observar que o domínio de Organização do Conhecimento (doravante OC) denominado Sistemas de Informação e Recuperação [Information Systems and Retrieval - IS\&R] representa sucessivamente nas três amostras 32.4 \% em 1965, 26.2 \% em 1975, voltando a crescer para 29.2 \% em 1985. Isso significa que nos anos 1960 e 1980 este domínio representou a maior área temática de sua amostra. Ainda neste domínio IS\&R, o tópico Recuperação da Informação [Information Retrieval] além de constante crescimento (4.2, 7.8 e $12.7 \%$ ) foi o maior item individual de pesquisa na década de 1980. O item de pesquisa Classificação e Indexação [Classification and Indexing] pertencente ao mesmo domínio, chegou a representar sozinho, mais de $20 \%$ dos artigos publicados, tendo representado $21.8 \%$ na década de $1960,13.6 \%$ na década de 1970 , caindo finalmente para $5.6 \%$ na década de 1980 . Observando-se ainda que as "populações" de artigos estudados cresceu em termo absolutos (de 142 em 1965 para 449 em 1985), observa-se que o número absoluto de artigos não teve um decréscimo tão significativo, pois se na amostra de 1965 foram produzidos cerca de 30 artigos, na amostra de 1985 foram produzidos cerca de 25 artigos. Houve portanto um grande crescimento da área de CI com um pequeno decréscimo na produção absoluta sobre OC e uma marcante diminuição relativa.

O quadro classificatório de Jarvelin e Vakkari (1993), inspirou Cano (1999), a realizar também um estudo bibliométrico da BCI (LIS) na literatura espanhola a partir da produção total de dois grandes periódicos (Revista Espanbola de Documentación Cientifica e a Documentación de las Ciencias de la Información), no período de 1977-1994. Trabalhando com um total de 354 artigos, o autor observou que, de modo geral, "como toda literatura de L \& IS, na Espanha parece ser igualmente distribuídos entre os artigos que abordam atividades de serviços L \& IS (ou BCI), Armazenamento e Recuperação da Informação e Estudos de Comunicação Científica e Profissional” (Cano, 1999: 677-678) como termos / palavras / conceitos mais pesquisados. O exame individual da distribuição dos temas por revista mostra uma distribuição diferente dos tópicos apresentados pelos dados agregados, e tal fato é consequência, segundo o autor, provavelmente, da diferença nas metas e objetivos do CINDOC - editor da Revista Espanbola de Documentación Cientifica - uma 
instituição do governo espanhol responsável pela divulgação de informação científica e técnica em todo o país e do Departamento de Documentação da Universidad Complutense de Madrid - editor da Documentación de las Ciencias de la Información - que reflete os interesses de um currículo escolar, localizado dentro do maior departamento de estudos da comunicação do país (Cano, 1999: 677-678).

Interessado no movimento de "acesso aberto" (open access-OA), Mukherjee (2009) investigou o impacto da LIS em periódicos eletrônicos de OA e analisou 17 periódicos eletrônicos de acesso totalmente abertos, publicados ininterruptamente durante o período 2000-2004, em termos de artigos, autores, instituições, países, assuntos e referências citadas. Um ponto negativo a ser mencionado sobre esse artigo, é que o autor sugere ter identificado o núcleo de periódicos eletrônicos em OA da LIS, entretanto, essa afirmação pode ser questionada, pois os periódicos analisados pelo autor possuem uma grande dispersão geográfica (o estudo inclui periódicos da África do Sul e da China), mas não são citados periódicos latino-americanos ou, sequer, ibéricos, fato que nos causa estranheza, pois nos perguntamos por que o estudo realizado por um autor espanhol não os considerou.

Neste estudo, as palavras-chave dos títulos foram classificadas em quatro níveis, sendo utilizadas as descrições da abrangência de assuntos do Jita Plano de Classificação de Biblioteconomia e Ciência da Informação. ${ }^{1}$ Os resultados demonstram que o assunto predominante foi a Tecnologia da Informação (308 artigos), seguido de Fontes de Informação (245 artigos) e o terceiro assunto mais frequente foi Tratamento da Informação para os Serviços de Informação (185). Outros temas de marcante interesse nesta amostra foram Tecnologia de Computadores para Biblioteca, Bibliotecas Digitais, Aplicações de TI (169 artigos), Conhecimento e Aprendizagem (124 artigos) (Mukherjee, 2009: 187-189).

Outro autor que se dedicou aos estudos métricos mais recentemente foi Aharony (2011), que analisou os periódicos top 10 na BCI de 2007-2008 segundo uma lista que inclui os 10 periódicos mais citados no período, pelo seu fator de impacto segundo o Journal Citation Report (JCR) de 2008 e refletindo um fator de impacto médio de 1,57. Dos 10 periódicos selecionados, ${ }^{2}$ seis são revistas de Ciência da Informação e quatro são revistas de graduação em BCI. A investigação estatística descritiva realizada em 1250 artigos foi

1 http://eprints.rclis.org/cms/jita

2 Os periódicos da lista "top 10" são: Journal of the American Society for Information Science and Technology, Scientometrics, Journal of Information Processing E Management, Journal of Computer-Mediated Communication, Journal of Information Science, Journal of Documentation, Information Research, College \& Research Libraries, Library \& Information Science Research, Journal of Global Information Management. 
seguido por uma análise de conteúdo aprofundada em 417 artigos (33\%). A análise de conteúdo com 856 palavras-chaves foi baseada no esquema de classificação da Ciência da Informação de Zins (2007). O resultado mostra que $17.99 \%$ das palavras-chave estão associadas com a tecnologia da informação, seguidas por 17.40 \% da metodologia, o Aspecto Social da Ciência da informação responsável por $17.05 \%$, e a organização de dados e recuperação por palavras-chave de $12.50 \%$ (Aharony, 2011: 30-31).

O último trabalho examinado foi o relatório produzido pelo Instituto Brasileiro de Informação em Ciência e Tecnologia (IBICT). Trata-se de um mapeamento de números temáticos indexados em bases de dados internacionais da área de BCI (Information Science \& Technology Abstracts - ISTA, Library, Information Science \& Technology Abstracts with Full Text - LISTA/ FULL e Library and Information Science Abstracts - LISA), no período de 2005-2010, disponíveis no Portal de Periódicos da Capes. A análise de conteúdo foi baseada em 185 números temáticos publicados em periódicos (themed issue; special issue) e 77 trabalhos na categoria painéis apresentados em 2 congressos da American Society for Information Science and Technology (ASIS\&T), de 2008 e 2009, e distribuídos em 11 categorias do Information Science Taxonomy (Hawkins, Larson e Caton, 2003). O resultado demonstrou que as categorias de Pesquisa em Ciência da Informação, Bibliotecas e Serviços Bibliotecários e Questões Sociais contem os assuntos mais pesquisados em periódicos e congressos. Já as categorias de Tecnologias da Informação, Indústria da Informação, Informação e Questões Governamentais e Legais, e Organização do Conhecimento possuem grandes diferenças entre as publicações em periódicos e congressos.

Quadro 1. Variações no Monitoramento internacional da produção científica em Cl

\begin{tabular}{|c|c|c|c|c|c|}
\hline \multirow[b]{2}{*}{ Categorias } & \multicolumn{2}{|c|}{ Periódicos } & \multicolumn{2}{|c|}{ Congresso } & \multirow{2}{*}{$\begin{array}{c}\text { Totais } \\
N^{0} \text { nas } \\
\text { categorias }\end{array}$} \\
\hline & $\begin{array}{c}\mathrm{N}^{0} \text { na } \\
\text { categoria }\end{array}$ & $\begin{array}{c}\% \text { Acumulado } \\
\text { na faixa }\end{array}$ & $\begin{array}{c}\mathrm{N}^{0} \text { na } \\
\text { categoria }\end{array}$ & $\begin{array}{c}\% \text { Acumulada } \\
\text { na faixa }\end{array}$ & \\
\hline $\begin{array}{l}\text { Pesquisa em Ciência da } \\
\text { Informação }\end{array}$ & $37(20 \%)$ & $20 \%$ & $31(40 \%)$ & $40 \%$ & $68(26 \%)$ \\
\hline $\begin{array}{l}\text { Bibliotecas e Serviços } \\
\text { Bibliotecários }\end{array}$ & $32(17 \%)$ & $37 \%$ & $12(16 \%)$ & $56 \%$ & $44(17 \%)$ \\
\hline Questões Sociais & $26(14 \%)$ & $51 \%$ & $12(16 \%)$ & $72 \%$ & $38(15 \%)$ \\
\hline Tecnologias da informação & $26(14 \%)$ & $65 \%$ & $6(7.5 \%)$ & $79,5 \%$ & $32(12 \%)$ \\
\hline Indústria da Informação & $17(9 \%)$ & $74 \%$ & $3(4 \%)$ & $83,5 \%$ & $20(7.6 \%)$ \\
\hline $\begin{array}{l}\text { Informação e Questões } \\
\text { Governamentais e Legais }\end{array}$ & $17(9 \%)$ & $83 \%$ & $3(4 \%)$ & $87,5 \%$ & $20(7.6 \%)$ \\
\hline $\begin{array}{l}\text { Organização do } \\
\text { Conhecimento }\end{array}$ & $12(6 \%)$ & $89 \%$ & $6(7.5 \%)$ & $95 \%$ & $18(7 \%)$ \\
\hline
\end{tabular}




\begin{tabular}{|l|c|c|c|c|c|}
\hline $\begin{array}{l}\text { Produção Editorial e } \\
\text { Distribuição }\end{array}$ & $8(4 \%)$ & $93 \%$ & -- & -- & $8(3 \%)$ \\
\hline Profissão da Informação & $3(2 \%)$ & $95 \%$ & $3(4 \%)$ & $99 \%$ & $6(2 \%)$ \\
\hline $\begin{array}{l}\text { Fontes e Aplicações para } \\
\text { Assuntos Específicos }\end{array}$ & $4(3 \%)$ & $98 \%$ & -- & -- & $4(1.5 \%)$ \\
\hline $\begin{array}{l}\text { Sistemas e Serviços de } \\
\text { Informação Eletrônica }\end{array}$ & $3(2 \%)$ & $100 \%$ & $1(1 \%)$ & $100 \%$ & $4(1.5 \%)$ \\
\hline
\end{tabular}

Fonte: 0 autor, baseado em IBICT (2011, v.1 e v.2)

A observação das seis pesquisas analisadas até aqui permite afirmar que houve uma mudança de foco da BCI, principalmente com os temas da Organização do Conhecimento (OC), decrescendo de foco principal para médio. Esse decréscimo nas pesquisas poderia ser decorrente das classificações que foram utilizadas, por isso, reproduzimos no quadro a seguir um comparativo dos termos utilizados para descrever a parte/subárea do domínio de OC em todos os estudos utilizado pelos autores aqui citados. Em todas as categorias utilizadas nos estudos relatados, vemos que um campo/área/domínio como a BCI (ou LIS) é constituído por temáticas, que podem ser analisadas como partes.

Quadro 2. Esquemas de Classificação de assunto na $\mathrm{Cl}$

\begin{tabular}{|c|c|c|c|}
\hline \multicolumn{4}{|c|}{ Esquemas de classificação de assuntos } \\
\hline $\begin{array}{c}\text { Jarvelin e Vakkari } \\
\text { (1993) }\end{array}$ & \begin{tabular}{|c|} 
Jita Plano de Classifi- \\
cação de Biblioteconomia \\
e Ciência da Informação \\
\end{tabular} & $\begin{array}{l}\text { Zins' classification } \\
\text { scheme (2007) }\end{array}$ & $\begin{array}{c}\text { Hawkins, Larson e Caton } \\
\text { (2003) }\end{array}$ \\
\hline $\begin{array}{l}50 \text { Information Searching } \\
\text { \&Retrieval } \\
\text { Study on... } \\
\text { 51. Cataloguing } \\
\text { 52. Classification and } \\
\text { indexing (process or } \\
\text { languages) } \\
\text { 53. Information retrieval } \\
\text { 54. Bibliographic databa- } \\
\text { ses or bibliographies } \\
\text { 55. Nonbibliographic } \\
\text { data bases (textual, } \\
\text { numeric...) }\end{array}$ & $\begin{array}{l}\text { Information treatement } \\
\text { for information } \\
\text { services } \\
\text { IA. Cataloguing, biblio- } \\
\text { graphic control. } \\
\text { IB. Content analysis. } \\
\text { IC. Index languages, } \\
\text { processes and } \\
\text { schemes. } \\
\text { ID. Data and metadata } \\
\text { structures. } \\
\text { IE. Knowledge represen- } \\
\text { tation. } \\
\text { IF. Information transfer: } \\
\text { protocols, formats, } \\
\text { techniques. } \\
\text { IG. Information presen- } \\
\text { tation: hypertext, } \\
\text { hypermedia. } \\
\text { IH. Image systems. } \\
\text { II. Filtering. }\end{array}$ & $\begin{array}{l}\text { 5. Data Organization } \\
\text { and Retrieval } \\
\text { - classification } \\
\text { schemes, } \\
\text { - metadata, } \\
\text { - indexing, } \\
\text { - text mining, } \\
\text { - abstracting, } \\
\text { - knowledge } \\
\text { organization, } \\
\text { - taxonomies, } \\
\text { - thesauri, } \\
\text { - ontology, } \\
\text { - vocabulary control, } \\
\text { - online searching } \\
\text { techniques, } \\
\text { - reference work, } \\
\text { - the semantic web. }\end{array}$ & $\begin{array}{l}\text { 2. Organização do conhe- } \\
\text { cimento } \\
\text { 2.1 Tesauros, listas de } \\
\text { autoridade Taxono- } \\
\text { mias, ontologias, } \\
\text { redes semânticas, } \\
\text { nomenclaturas, } \\
\text { terminologias, } \\
\text { vocabulários. } \\
\text { 2.2 Catalogação e } \\
\text { classificação } \\
\text { Tags, metatags, } \\
\text { metadados, Dublin } \\
\text { Core (Dublin Core } \\
\text { Metadata Initiati- } \\
\text { ve-DCMI), identifi- } \\
\text { cadores de objetos } \\
\text { digitais (digital object } \\
\text { identifiers-DOls), ca- } \\
\text { tálogos para acesso } \\
\text { público em linha }\end{array}$ \\
\hline
\end{tabular}




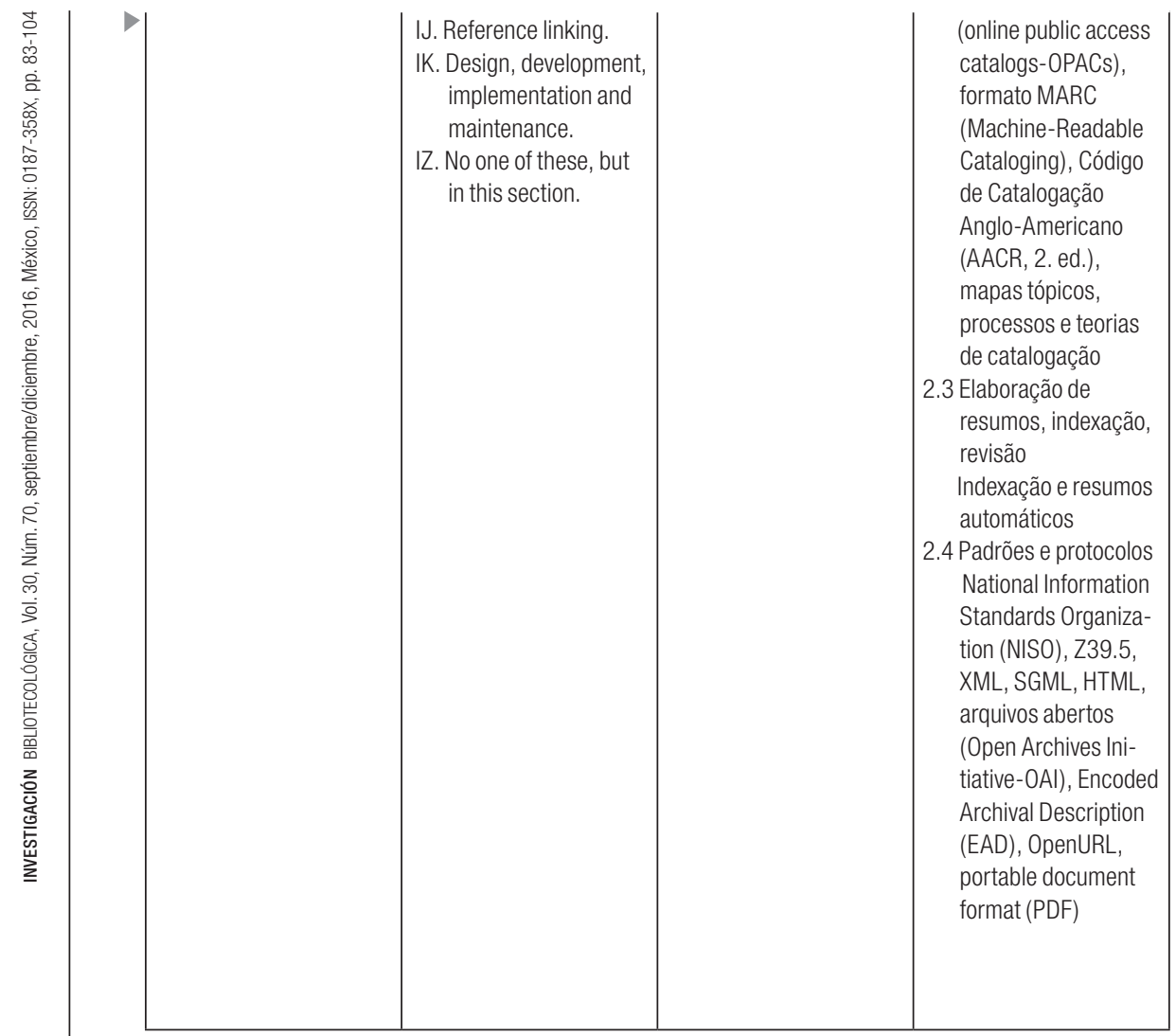

Fonte: 0 autor, baseado em Jarvelin e Vakkari (1993); Jita Plano de Classificação de Biblioteconomia e Ciência da Informação (http://eprints.rclis.org/cms/jita); Aharony (2011: 29-30); IBICT (2011, v.1 e v.2)

Os estudos realizados por Jarvelin e Vakkari (1993), Cano (1999), Mukherjee (2009), Aharony (2011) e IBICT (2011, v.1 e v.2) demonstraram uma tendência de diminuição numérica relativa de ocorrências de artigos discorrendo sobre o domínio da Organização do Conhecimento na área de Ciência da Informação, e esta diminuição relativa passa a constituir um problema quando a confrontamos com o discurso de Hjørland (1998: 610) quando este afirma que análise de assunto dos documentos (Organização do Conhecimento) é a atividade mais importante feita pelos bibliotecários e profissionais de informação.

Assim, propusemos como objetivo geral analisar comparativamente a ocorrência numérica absoluta e relativa de artigos de periódicos brasileiros da área de Ciência da Informação (CI) sobre a temática Organização do Conhecimento (OC) cobertos na Base Referencial de Artigos de Periódicos em 
Ciência da Informação (BRAPCI) ${ }^{3}$ de 1972 a 2012 através de recortes temático-conceituais.

Os objetivos específicos decorrentes do objetivos geral foram: a) identificar a ocorrência numérica relativa e absoluta dos artigos de periódicos relativos à organização do conhecimento através de recortes temático-conceituais e b) identificar dentre os periódicos da CI brasileiros o núcleo mais devotado à OC.

\section{Metodologia}

Esta pesquisa é descritiva e quantitativa, utilizando de métodos bibliométricos que se inserem nas metodologias estatísticas de coleta e análise de dados. Utilizado ainda da classificação de Fonseca (1986: 10-11), podemos adicionar o caráter especializado ou microbibliométrico dessa pesquisa dado ao seu objeto de estudo.

A opção pelo uso de metodologias quantitativas se deu ainda porque possibilitou investigar "a extensão na qual as variações em um fator correspondem às variações em um ou mais fatores, baseados em coeficientes de correlação" (Preti, 2006: 13).

A aplicação desses métodos quantitativos às bases de dados permite meta-análises por reunir e (re)combinar "[...] os resultados de outros estudos realizados de forma independente (geralmente extraídos de trabalhos publicados) e sintetizar suas conclusões ou mesmo extrair uma nova conclusão" (Luiz, 2002: 409). Para o mesmo autor uma meta-análise permite uma reflexão crítica (Luiz, 2002: 410).

Ainda segundo Flick (2004, em Braga, 2007: 27) as análises quantitativas possibilitariam, provavelmente em momento posterior e supomos, via análise qualitativa "isolar claramente causas e efeitos, operacionalizar corretamente relações teóricas [...]”.

Deste modo, ainda que se recorra a métodos quantitativos, podemos considerar que essa pesquisa tem um aporte analítico-qualitativo, uma vez que

3 BRAPCI é o resultado do projeto de pesquisa Opções metodológicas em pesquisa: a contribuição da área da informação para a produção de saberes no ensino superior, sendo mantida pelo Grupo de pesquisa Educação, Pesquisa e Perfil Profissional em Informação (E3PI) da Universidade Federal do Paraná (UFPR). Sendo um projeto financiado pelo CNPq, A base BRAPCI vem sendo utilizada para pesquisas de tendências epistemológicas através da análise dos movimentos de produção sobre certos temas na Ciência da Informação, por exemplo, pelo Grupo de Pesquisa Informação, Discurso e Memória, coordenado pela professora Lídia Silva de Freitas. Desde sua tese de doutorado apresentada à ECA/USP, desenvolveu uma metodologia de recortes temático-discursivos que, na época, foi aplicada a dois periódicos. Atualmente a Base é composta por 37 periódicos nacionais impressos e eletrônicos da área de CI, 26 estão ativos e 11 históricos (descontinuados). 
as questões levantadas nos objetivos específicos da pesquisa foram formuladas no sentido de entender uma série de fenômenos, dentre eles: o surgimento e o envelhecimento dos temas de pesquisa atinentes à Organização do Conhecimento, quem são os principais autores brasileiros.

As pesquisas quali-quantitativas, se por um lado, traduzem em números as opiniões e informações para serem analisadas e classificadas através da produção de tabelas e gráficos estatísticos (Cordeiro, 2012: 30), por outro lado, permitem inferências analíticas de nível qualitativo em geral feitos a partir do levantamento dos dados. Para realizarmos esta modalidade híbrida de pesquisa, percorremos as seguintes etapas.

\section{Levantamento de conceitos ligados à Organizaçâo do Conbecimento nos ENANCIBs}

O levantamento dos conceitos da OC foi realizado sem o uso de motores de busca nos anais disponíveis no Portal da $\mathrm{ANCIB}^{4}$ dos anos de 2003 a 2009 e nos endereços dos sites que hospedaram o evento nos anos de $2010,{ }^{5} 2011^{6}$ e $2012 .^{7}$

Foram levantados e incluídos os conceitos atribuídos como palavras chaves pelos autores que publicaram nos Grupos de Trabalhos 2 e 8 dos Encontros Nacionais de Pesquisa em Ciência da informação - ENANCIBs, considerando os critérios de avaliação desses encontros, que os caracterizam como "superformais" e que estes representariam a frente de pesquisa no Brasil (Braga, 1973). Nas palavras de Salek (2011: 57): " a comparação entre as frequências das categorias analíticas permite observar que os trabalhos dos ENANCIBs apresentam uma aparente maior 'cautela' na adesão a modismos e/ou noções com fraca fundamentação teórica”.

A justificativa para uma aparente inversão da qualidade científica entre as duas fontes, que transparece na utilização ou não de terminologia e conceitos científicos e na adesão ou não a modismos pouco aprofundados pode estar na dupla orientação do campo - voltado 'à explicação e à intervenção nos processos humanos de conhecimento, memória, comunicação’. (González de Gómez, 2000 em Salek, 2011: 57).

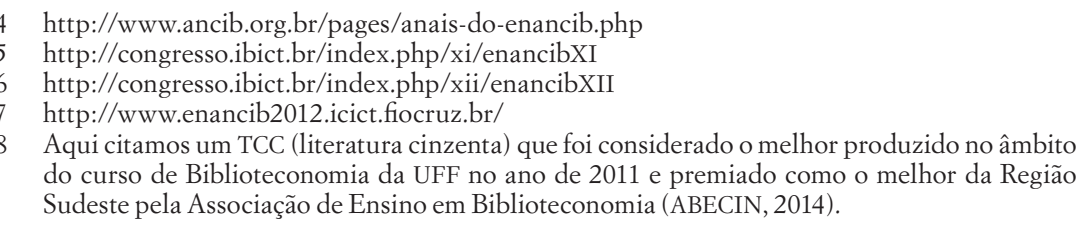


A superformalidade de avaliação pelos pares no ENANCIB legitimaria os relatos de pesquisa concluídos (apresentados oralmente) ou em desenvolvimento (apresentados em formato de pôsteres) como representativos da frente de pesquisa brasileira neste domínio. Do mesmo modo, essa avaliação garantiria a legitimidade de reificação dessas temáticas como objeto de pesquisa neste domínio.

Os sinais de separação entre os conceitos, tais como ponto, virgula ou ponto e virgula, não foram considerados como sinais de pré-coordenação. De modo contrário, termos unidos por hífen foram analisadas como um único conceito. Segundo Lancaster (2004, cap. 4) a hifenização de termos para construção de índices pré-coordenados é uma escolha arbitrária do indexador, com vistas a representar um assunto unidimensionalmente. ${ }^{9}$

Ainda quanto à pré-coordenação de termos, nos casos em que foram encontrados conceitos correspondentes, estes foram computados como único conceito. Por exemplo: Representação da informação e Representação - Informação.

Também adotamos esse critério de equivalência para conceitos grafados em outras línguas, sendo assim, foram computados junto com seus correspondentes em português no singular, por exemplo: Thesaurus ou Tesauros, formam computados como Tesauro. Outro critério observado para nove conceitos específicos foi adequá-los à forma ortográfica com preposições diferentes (de ou da), no plural, no singular e acrônimos (siglas), para que fossem computados na forma como a maioria dos autores utiliza nos descritores ou palavras-chave dos seus trabalhos nos ENANCIBs.

Fo excluídos da análise 17 artigos não apresentam palavras-chave.

Assim, a extração de conceitos foi realizada a partir de uma amostra de 376 trabalhos publicados nos GT 2 e GT $8 .{ }^{10}$ A partir das palavras-chaves foram identificados 919 conceitos dos quais somente 238 foram considerados pertencentes ao domínio da OC. A falta de uniformidade dos conceitos falseia o número de ocorrências, pois as flexões nominais e numéricas fazem conceitos iguais aparecerem com ocorrências diferentes, de modo que estas diferentes formas de enunciação estão apresentadas, portanto, de forma "bruta" não servindo para uma posterior análise.

9 Ainda segundo Lancaster (2004: 52-53) para que um índice pré-coordenado permita mais que uma dimensão na representação é necessário um processo de alternação, rotação, ou deslocamento dos termos ou conceitos.

10 O Gráfico 2 demonstra a variação da quantidade do número de trabalhos publicados por anos nesses GTs. Devido à falta de espaço será apresentado como documento suplementar. 


\section{Acesso à Base BRAPCI e identificação e extração dos dados da base}

A base empírica para essa dissertação foi formada a partir da seleção de artigos de periódicos brasileiros indexados na Base BRAPCI.

Cada conceito mapeado foi pesquisado através do mecanismo de busca utilizando aspas ("”) $)^{11}$ limitando-se ainda a busca apenas nas palavras-chave. A razão para trabalharmos apenas sobre o campo de busca das palavras-chaves é que os termos indexados representariam a centralidade das enunciações das temáticas principais dos artigos na visão dos próprios autores.

\section{Construção de planilhas para análise de dados}

Após o acesso e consulta aos artigos, as palavras-chave atribuídas pelos autores foram extraídas, exportadas e arranjadas em planilha eletrônica (software Excel).

A seleção dos artigos foi realizada a partir do seguinte parâmetro:

- abrangência da área de assunto claramente exposta nas palavras-chave, permitindo identificação e enquadramento nos termo da área temática da pesquisa: OC;

A visualização destes elementos apresentados em todos os registros recuperados pela base fez necessária uma arrumação de forma a serem trabalhados de acordo com nossa necessidade. Os registros na BRAPCI foram (re) formatados em planilha Excel, conforme o Quadro 3:

Quadro 3. Modelo utilizado para reformatar dados em planilha Excel

\begin{tabular}{|c|l|l|l|c|c|l|}
\hline Número & \multicolumn{1}{|c|}{ Título } & \multicolumn{1}{|c|}{ Autor } & Periódico & Ano & Seção & Palavras-chave \\
\hline 1 & $\begin{array}{l}\text { Construção de lingua- } \\
\text { gens documentárias } \\
\text { em sistemas de } \\
\text { recuperação da infor- } \\
\text { mação: a importância } \\
\text { da garantia do usuário }\end{array}$ & $\begin{array}{l}\text { Oliveira, Dal- } \\
\text { giza Andrade; } \\
\text { Araújo, Ronal- } \\
\text { do Ferreira de }\end{array}$ & $\begin{array}{l}\text { Encontros } \\
\text { Bibli: Revista } \\
\text { Eletrônica de } \\
\text { Biblioteconomia } \\
\text { e Ciência da } \\
\text { Informação }\end{array}$ & 2012 & Artigo & $\begin{array}{l}\text { Garantia do usuário; } \\
\text { Organização do con- } \\
\text { hecimento; Tesauros; } \\
\text { Representações } \\
\text { documentárias; } \\
\text { Indexação }\end{array}$ \\
\hline
\end{tabular}

11 Assim como os operadores booleanos (and, or, not), as aspas são comando padrão universal em mecanismos de busca para recuperar informação na forma exata que se deseja. Na própria BRAPCI é informado na tela principal, abaixo da caixa de busca, que para termos compostos as aspas deve ser usadas. 
Após a coleta e (re)formatação padronizada dos dados, realizamos um estudo comparativo que nos possibilitou mensurar, analisar e diagnosticar qual é a importância relativa da Organização do Conhecimento na Ciência da Informação no Brasil, mas especificamente quem escreve, quais tipos de material são mais utilizados, em quais periódicos mais pública, entre outras características.

É valido ressaltar que a todo o momento adotamos a posição de simples consulentes ou usuários das plataformas web, de maneira que não mantivemos nenhum tipo de contato com os gestores do Portal da ANCIB ou da base BRAPCI.

\section{Resultados}

Das 238 palavras-chave pertinentes ao domínio da Organização do Conhecimento extraídas dos trabalhos no GT2 e 8 dos anos de 2003 a 2012, apresentados na seção 2.5, 127 (53.4 \%) apresentaram resultados ao serem pesquisados na BRAPCI, os outros 111 (46.6\%) não tiveram nenhuma incidência de documentos.

Merecem destaque ainda que trabalhos recuperados por quatro conceitos não foram contabilizados na análise, pois iriam comprometer os resultados, são eles: "Conceito", "Relacionamento", "Representação" e "Informação - Representação”. O primeiro apresentou um número de documentos em sua grande maioria tratando de epistemologia, como exemplos, o "conceito de informação na Ciência da Informação" ou "conceito de biblioteca" etc. Todos os documentos indexados pela base por "Conceito" que pertenciam ao domínio da OC foram indexados também com outros descritores mais específicos, portanto, já estando cobertos na análise.

Já o conceito de "Relacionamento" só apresentou resultados abordando os fatores psicológicos, como exemplo: entre bibliotecários e usuários, entre unidades de informação e seus clientes etc.

O termo e conceito de "Representação", por ser muito genérico, também apresentou um número considerável de trabalhos abordando a representação social de indivíduos em grupos minoritários, da mesma forma, seus trabalhos não foram contabilizados para não comprometerem os resultados dessa pesquisa. Cabe ressaltar que antes de tomarmos essa decisão foi feita pesquisa sobre os conceitos de "Representação descritiva" e "Representação temática”, pois ambos são foco da OC. Em ambos as conceitos, os artigos recuperados já estavam cobertos na análise, sendo indexados por outros conceitos como exemplo "Catalogação" e "Tesauro". 
A relação de termos "Informação - Representação" recuperou apenas um trabalho na seção Temas da revista Transinformação. O trabalho de Jardim, José Maria, "Informação e representação social”, Transinformação 8 (1) (jan./ abr.), 1996, que discute a representação social de um sujeito a partir de seu papel de agente propagador de informações, por não se tratar de Organização do Conhecimento, fez com que o mesmo não fosse contabilizado.

Após a reformatação ainda foi necessário um processo de deduplicação a fim de eliminar dados de artigos com mais de uma ocorrência, em que foi possível identificar a republicação de 3 artigos, todos contabilizados uma única vez.

Ao final, alcançamos o total de 750 artigos no período de 1972 a 2012, sobre os quais faremos nossas considerações a seguir.

\section{Produção relativa sobre organização do conhecimento}

Este primeiro resultado diz respeito ao objetivo "a".

Considerando o total de documentos incluídos na base no período 19722012 (7 754) e o total de documentos sobre Organização do Conhecimento no mesmo período, 750, chegamos ao percentual de $9.7 \%$ para responder, então, ao nosso objetivo “a”. Este percentual é muito próximo dos resultados no Monitoramento internacional da produção científica em CI realizado pelo IBICT (2011, v.1 e v.2) no qual chegou a um resultado de 7 \% somando os trabalhos publicados em periódicos e congressos.

Especificamente sobre o domínio da Organização do Conhecimento observamos que o mesmo se encontra em expansão.

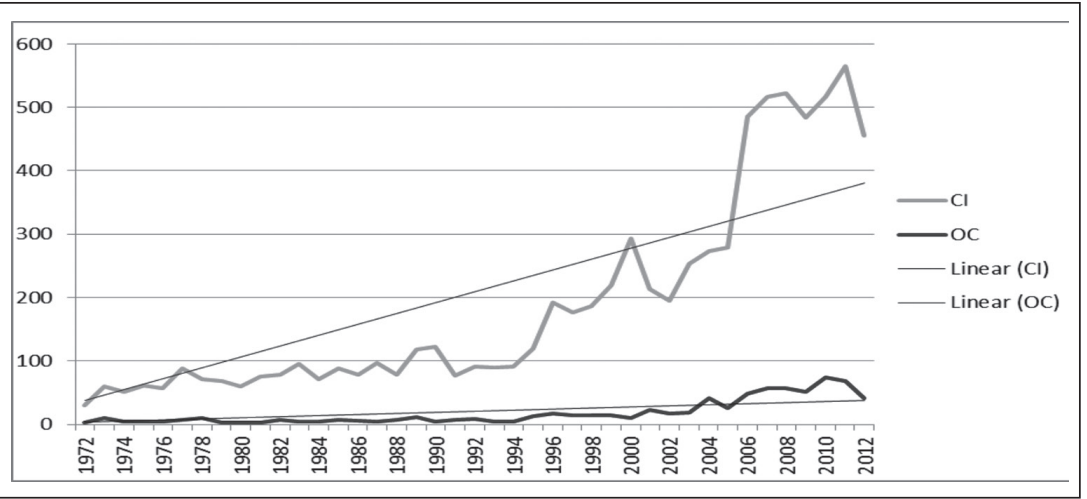

Gráfico 1. Comparativo entre o Domínio da OC e a Cl

Fonte: 0 autor. 
No quadro a seguir observamos que em um período de seis anos (2006 a 2012) foram produzidos mais da metade do total de artigos concernentes ao domínio da OC do que todo o período de trinta e três anteriores (1972 a 2005).

Quadro 4. Produtividade relativa entre $\mathrm{OC} \mathrm{e} \mathrm{Cl}$

\begin{tabular}{|c|c|c|c|c|c|c|c|}
\hline Ano & $\mathrm{Cl}$ & $\%$ & $\sum \%$ & OC & $\%$ & $\sum \%$ & $\mathrm{OC} / \mathrm{Cl}$ \\
\hline 1972 & 30 & 0.4 & 0.4 & 3 & 0.4 & 0.4 & 10 \\
\hline 1973 & 60 & 0.77 & 1.17 & 11 & 1.46 & 1.86 & 18.33 \\
\hline 1974 & 52 & 0.67 & 1.84 & 5 & 0.67 & 2.53 & 9.61 \\
\hline 1975 & 62 & 0.8 & 2.64 & 4 & 0.53 & 3.06 & 6.45 \\
\hline 1976 & 57 & 0.74 & 3.38 & 4 & 0.53 & 3.59 & 7.02 \\
\hline 1977 & 88 & 1.13 & 4.51 & 8 & 1.07 & 4.66 & 9.09 \\
\hline 1978 & 72 & 0.93 & 5.44 & 10 & 1.33 & 5.99 & 13.88 \\
\hline 1979 & 69 & 0.89 & 6.33 & 3 & 0.4 & 6.39 & 4.35 \\
\hline 1980 & 60 & 0.77 & 7.1 & 3 & 0.4 & 6.79 & 5 \\
\hline 1981 & 76 & 0.98 & 8.08 & 3 & 0.4 & 7.19 & 3.94 \\
\hline 1982 & 79 & 1.02 & 9.1 & 8 & 1.07 & 8.26 & 10.13 \\
\hline 1983 & 95 & 1.23 & 10.33 & 5 & 0.67 & 8.93 & 5.26 \\
\hline 1984 & 72 & 0.93 & 11.26 & 4 & 0.53 & 9.46 & 5.56 \\
\hline 1985 & 89 & 1.14 & 12.4 & 8 & 1.07 & 10.53 & 8.98 \\
\hline 1986 & 78 & 1.01 & 13.41 & 6 & 0.8 & 11.33 & 7.69 \\
\hline 1987 & 97 & 1.25 & 14.66 & 5 & 0.67 & 12 & 5.15 \\
\hline 1988 & 78 & 1.01 & 15.67 & 7 & 0.93 & 12.93 & 8.97 \\
\hline 1989 & 119 & 1.53 & 17.2 & 12 & 1.6 & 14.53 & 10.08 \\
\hline 1990 & 123 & 1.59 & 18.79 & 4 & 0.53 & 15.06 & 3.25 \\
\hline 1991 & 77 & 1 & 19.79 & 8 & 1.07 & 16.13 & 10.39 \\
\hline 1992 & 91 & 1.17 & 20.96 & 9 & 1.2 & 17.33 & 9.89 \\
\hline 1993 & 90 & 1.16 & 22.12 & 4 & 0.53 & 17.86 & 4.44 \\
\hline 1994 & 92 & 1.19 & 23.31 & 5 & 0.67 & 18.53 & 5.43 \\
\hline 1995 & 120 & 1.55 & 24.86 & 13 & 1.73 & 20.26 & 10.83 \\
\hline 1996 & 193 & 2.49 & 27.35 & 18 & 2.4 & 22.66 & 9.33 \\
\hline 1997 & 176 & 2.27 & 29.62 & 15 & 2 & 24.66 & 8.52 \\
\hline 1998 & 187 & 2.41 & 32.03 & 14 & 1.87 & 26.53 & 7.49 \\
\hline 1999 & 219 & 2.82 & 34.85 & 14 & 1.87 & 28.4 & 6.39 \\
\hline 2000 & 294 & 3.79 & 38.64 & 10 & 1.33 & 29.73 & 3.4 \\
\hline 2001 & 214 & 2.76 & 41.4 & 23 & 3.07 & 32.8 & 10.78 \\
\hline 2002 & 195 & 2.51 & 43.91 & 18 & 2.4 & 35.2 & 9.23 \\
\hline 2003 & 254 & 3.28 & 47.19 & 19 & 2.53 & 37.73 & 7.48 \\
\hline 2004 & 274 & 3.53 & 50.72 & 42 & 5.6 & 43.33 & 15.33 \\
\hline 2005 & 279 & 3.6 & 54.32 & 26 & 3.47 & 46.8 & 9.32 \\
\hline 2006 & 485 & 6.25 & 60.57 & 49 & 6.53 & 53.33 & 10.1 \\
\hline 2007 & 516 & 6.65 & 67.22 & 57 & 7.6 & 60.93 & 11.05 \\
\hline 2008 & 522 & 6.73 & 73.95 & 57 & 7.6 & 68.53 & 10.92 \\
\hline 2009 & 484 & 6.24 & 80.19 & 52 & 6.93 & 75.46 & 10.74 \\
\hline
\end{tabular}




\begin{tabular}{|c|c|c|c|c|c|c|c|}
\hline 2010 & 516 & 6.65 & 86.84 & 74 & 9.87 & 85.33 & 14.34 \\
\hline 2011 & 565 & 7.29 & 94.13 & 69 & 9.2 & 94.53 & 12.21 \\
\hline 2012 & 455 & 5.87 & 100 & 41 & 5.47 & 100 & 9.01 \\
\hline Total & 7754 & $100 \%$ & $100 \%$ & 750 & $100 \%$ & $100 \%$ & \\
\hline
\end{tabular}

Fonte: 0 autor

Os resultados do gráfico e do quadro anteriores contradizem os resultados do estudos realizados por Jarvelin e Vakkari (1993), Cano (1999), Mukherjee (2009), Aharony (2011) e IBICT (2011, v.1 e v.2) de que a Organização do Conhecimento estaria sofrendo um processo de encolhimento frente a outras temáticas.

Pelos dados da nossa tabela é possível ver que 53.2\% da produção brasileira sobre o domínio de OC na CI foi publicada entre 2006 e 2012, e que os demais $46.8 \%$ precisaram de 34 anos para serem produzidos sob a forma escrita. Observe-se ainda que se no ano de 1972 o domínio de OC representava $0.4 \%$ da produção de artigos de CI no Brasil, a partir do ano de 2006 chega a $6.53 \%$ e em 2011 chega a $9.2 \%$. O ano de 2012, mesmo que a cobertura não estivesse completa em abril de 2013 quando fechamos esse levantamento o domínio de OC já representava 5.47 \% na área. Inegavelmente, esses 5.47 \% significam que a OC decuplicou sua presença relativa na CI.

Considerando que o ano de 2012 não tinha ainda sua cobertura completa na base, podemos concluir que no Brasil, o domínio da OC ao contrário da tendência internacional está em relativa expansão mesmo frente a novas temáticas surgidas com os ambientes digitais (ou até por causa destes).

\section{Produtividade relativa: periódicos da ci mais devotados a OC}

Para atender ao nosso objetivo " $b$ ", devemos observar a produção de artigos de OC nos periódicos de CI. Onde dos 37 periódicos cobertos pela BRAPCI, 34 publicam artigos sobre a OC.

Observamos que a revista Ciência da Informação (139 artigos, $18.53 \%$ da amostra) é a mais produtiva em relação ao domínio de OC. Tal fato pode ser encarado como normal por ser a publicação mais antiga na área de CI no Brasil estando corrente atualmente.

A surpresa nesta observação, foi a revista DataGramaZero (86 artigos, $11.46 \%$ da amostra), que apesar de muito mais recente, tem um número considerável de publicações em OC, sendo inclusive superior aos números de publicações da revista Perspectivas em Ciência da informação (67 artigos, 8.93 \% da amostra). Devemos destacar ainda que somente o somatório dos resultados da Revista da Escola de Biblioteconomia da UFMG - precursora da Pers- 
pectiva - poderia superar o número de publicações da DataGramaZero, por uma diferença de apenas 7 artigos, o que incorreria em uma margem de erro de $0.9 \%$.

Em uma distribuição por zonas de produtividade podemos observar que as revistas Ciência da Informação e DataGramaZero, detém um terço (29.99 \%) das publicações, portanto, se constituindo como núcleo de publicação do domínio de OC no Brasil. As revistas Perspectivas em Ciência da Informação, Transinformação, Encontros Bibli e Revista de Biblioteconomia de Brasília detém outro terço (29.73 \%), portanto, se constituindo como zona transitória entre alta e baixa produtividade de publicações do domínio de OC no Brasil.

Consequentemente os 28 periódicos restantes se constituem como zona de dispersão das publicações sobre OC.

O Quadro 5 e o Gráfico 2 a seguir, demonstram essa observações da incidência de artigos de OC por periódico em números absolutos e percentuais.

Quadro 5. Produtividade de OC em periódicos

\begin{tabular}{|c|c|c|c|c|c|}
\hline Zonas & Rank & Frequência & $\%$ & $\sum \%$ & Titulo \\
\hline 1 & 1 & 139 & 18.53 & 18.53 & Ciência da Informação \\
\hline \multirow{2}{*}{2} & 2 & 86 & 11.46 & 29.99 & DataGramaZero \\
\hline & 3 & 67 & 8.93 & 38.92 & Perspectivas em Ciência da Informação \\
\hline \multirow{3}{*}{3} & 4 & 60 & 8 & 46.92 & Transinformação \\
\hline & 5 & 54 & 7.2 & 54.12 & Encontros Bibli \\
\hline & 6 & 42 & 5.6 & 59.72 & Revista de Biblioteconomia de Brasília \\
\hline \multirow{5}{*}{4} & 7 & 39 & 5.2 & 64.92 & Informação \& Informação \\
\hline & 8 & 33 & 4.4 & 69.32 & Informação \& Sociedade: Estudos \\
\hline & 9 & 30 & 4 & 73.32 & Revista Brasileira de Biblioteconomia e Documentação \\
\hline & 10 & 26 & 3.46 & 76.78 & Revista da Escola de Biblioteconomia da UFMG \\
\hline & 11 & 24 & 3.2 & 79.98 & Revista Digital de Biblioteconomia \& Cl \\
\hline \multirow{14}{*}{5} & 12 & 21 & 2.8 & 82.78 & Revista ACB: Biblioteconomia em Santa Catarina \\
\hline & 13 & 18 & 2.4 & 85.18 & Ponto de Acesso \\
\hline & 14 & 13 & 1.73 & 86.91 & BIBLOS \\
\hline & 15 & 12 & 1.6 & 88.51 & Biblionline \\
\hline & 16 & 12 & 1.6 & 90.11 & Em Questão: \\
\hline & 17 & 9 & 1.2 & 91.31 & Brazilian Journal of Information Science \\
\hline & 18 & 9 & 1.2 & 92.51 & Revista Ibero-Americana de Ciência da Informação \\
\hline & 19 & 8 & 1.06 & 93.57 & Informare: \\
\hline & 20 & 7 & 0.93 & 94.5 & Arquivistica.net \\
\hline & 21 & 7 & 0.93 & 95.43 & InCID: Revista de Ciência da Informação e Documentação \\
\hline & 22 & 5 & 0.66 & 96.09 & Liinc em revista \\
\hline & 23 & 4 & 0.54 & 96.63 & Cadernos de Biblioteconomia \\
\hline & 24 & 4 & 0.54 & 97.17 & Revista Eletrônica Informação e Cognição \\
\hline & 25 & 3 & 0.4 & 97.57 & Comunicação \& Informação \\
\hline
\end{tabular}




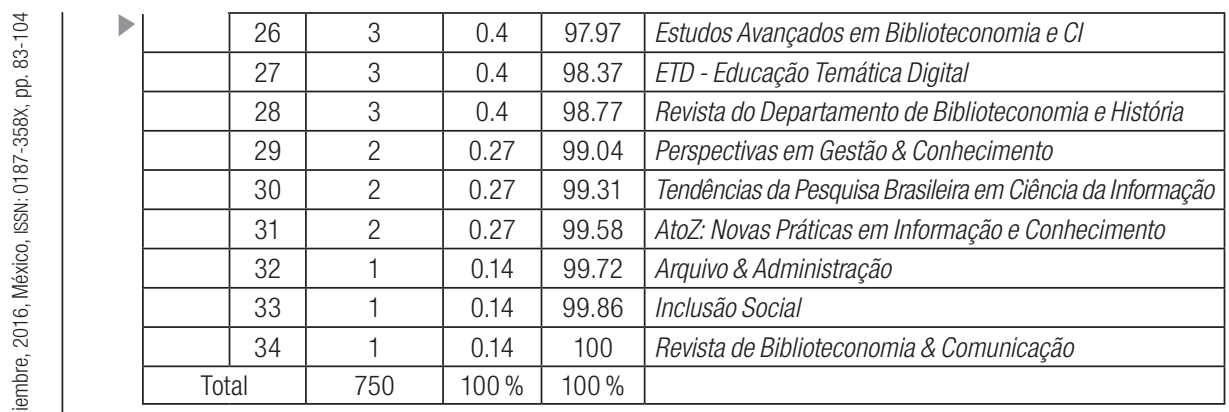

Fonte: Dados da pesquisa

Nos termos da Lei de Bradford, teríamos um periódico nuclear no domínio de OC, que sozinho publicou 139 artigos, formando a Z1 (zona 1), em seguida mais quatro zonas, cada uma com uma concentração numérica de artigos muito próxima da zona 1 ( $Z 2=153, Z 3=156, Z 4=152$ e Z5=150 artigos).

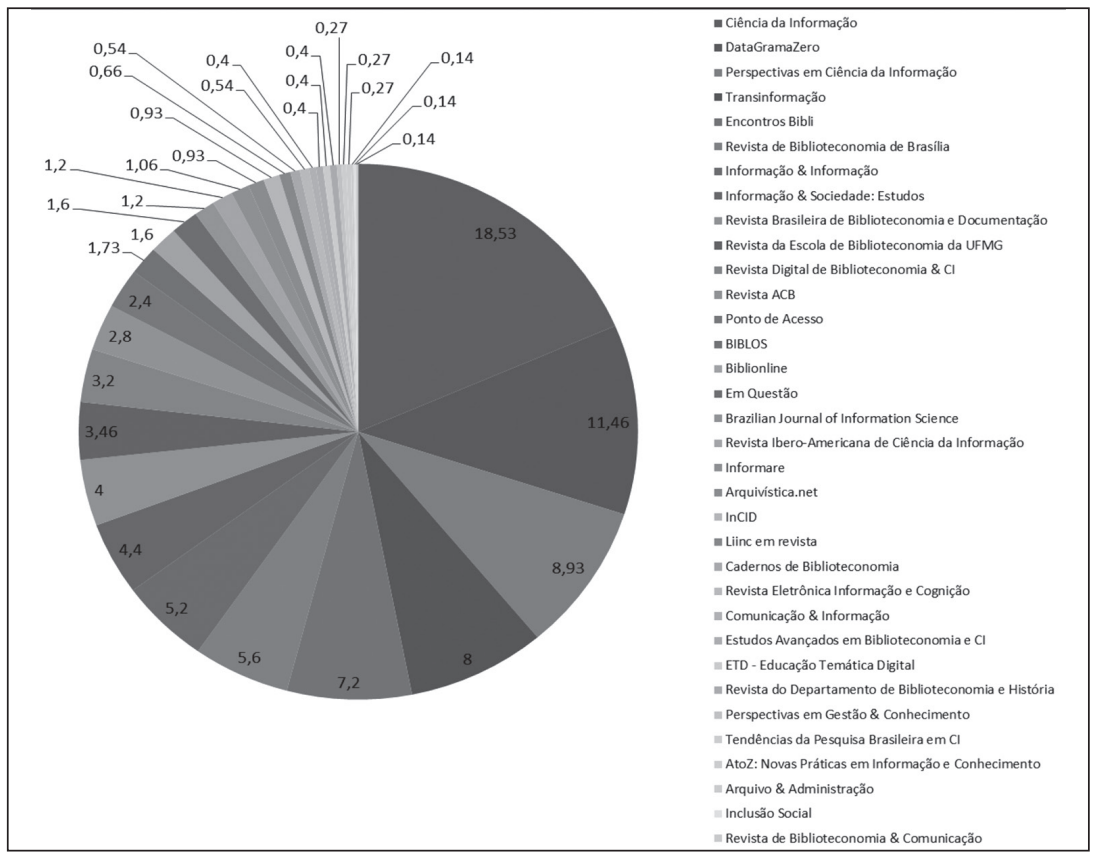

Gráfico 2. Incidência percentual de artigos de OC por periódico Fonte: Dados da pesquisa

Diante dessas descobertas passemos às nossas considerações finais. 


\section{CONSIDERAÇÕES FINAIS}

As questões levantadas permitiram caracterizar algumas práticas na produção das pesquisas sobre Organização do Conhecimento no Brasil e essa análise das práticas de produção escrita, feita a posteriori, sem dúvida, caracteriza como uma pesquisa qualitativa.

Primeiramente nosso mapeamento nos possibilitou identificar o domínio de Organização do Conhecimento a partir dos seus vários conceitos encontrados nas indexações presumivelmente autorais dos trabalhos do GT2 e GT8 publicados nos Anais dos ENANCIBs no período de 2003 a 2012. Esse passo foi descrito em nossa metodologia.

A partir deste pré-levantamento qualitativo, realizou-se um estudo quantitativo que nos possibilitou vislumbrar a produção brasileira no domínio de OC encontrada na base BRAPCI no período de 1972 a 2012, demonstrando que embora discreta em relação ao total da base, este domínio se encontra em crescimento. Isso foi demonstrado porque a média de publicações de 1972 a 2005 era em torno de 10.32 artigos por ano, ao passo que de 2006 a 2012 a média salta para 57 artigos por ano, representando uma alta maior que $500 \%$ (551\%). Como comentado no final da seção "Produção relativa sobre organização do conhecimento", foi constatado que $53.2 \%$ da produção brasileira sobre o domínio de OC na CI foi publicada entre 2006 e 2012 e que os demais $46.8 \%$ dos artigos foram publicados nos 34 anos anteriores. Observou-se também que no ano de 1972 o domínio de OC representava $0.4 \%$ da produção de artigos de CI no Brasil e a partir do ano de 2006 chegando a $6.53 \%$ e em 2011 chegando a $9.2 \%$. No ano de 2012, mesmo que a cobertura não estivesse completa em abril de 2013 o domínio de OC já representava $5.47 \%$ do total de artigos na CI. Inegavelmente, mesmo esse $5.47 \%$ em 2012 significam que a OC decuplicou sua presença relativa na CI nos últimos 34 anos, pois era $0.4 \%$ em 1972. Isso nos permite afirmar que no Brasil, contrariando a tendência internacional, a produção sobre OC está em crescimento relativo em relação à área de CI.

As observações decorrentes do nosso objetivo " $b$ " constataram que nem todos os periódicos cobertos pela base BRAPCI publicam sobre o domínio de OC. A surpresa nessa observação foi um periódico mais recente ter uma produtividade relativa superior a um periódico com mais anos de publicação, respectivamente a DataGramaZero e Perspectivas em Ciência da Informação. Também observamos que publicação de artigos em OC segue o modelo de dispersão da literatura de Bradford, no qual os periódicos mais devotados podem ser organizados por zonas de dispersão, sendo o mais devotado à OC a revista Ciência da Informação. 
Se por um lado, este artigo procurou ver o lugar epistemológico da Organização do Conhecimento na Ciência da Informação brasileira sob o aspecto da "moda" científica, seguindo os passos metodológicos indicados, consideramos ter sido possível responder à nossa questão de pesquisa, a qual permitiu observar que o domínio de Organização do Conhecimento, no Brasil, conforme demonstrado no Gráfico 1, encontra-se em crescente expansão. Este fato contraria análise de declínio com base nos mapeamentos realizados em âmbito internacional realizados por por Jarvelin e Vakkari (1993), Cano (1999), Mukherjee (2009), Aharony (2011) e IBICT (2011, v.1 e v.2).

Esta pesquisa não dá conta de todas as possibilidades de análise que poderiam ser feitas sobre o domínio da OC, por isso, nosso próximo passo são: os autores mais produtivos e a rede de colaboração entre os autores, o que com certeza revelará outras características como exemplo, o grau de colaboração entre as instituições e a instituição que possui maior incidência de pesquisadores de Organização do Conhecimento.

Deixamos assim um legado reflexivo e questões inseridas no âmbito da comunicação científica para nossas pesquisas não só sobre o domínio de OC, mas para todo o campo de CI no Brasil.

\section{REFERÊNCIAS}

Aharony, N. 2011. "Library and Information Science research areas: A content analysis of articles from the top 10 journals 2007-8". Journal of Librarianship and Information Science 44 (1): 27-35.

ABECIN (Associação Brasileira de Educação em Ciência da Informação). 2014. Concurso TCC: resultado 2012-2014. Londrina: ABECIN. http://www.abecin.org.br/abecin_conteudo.php?id=9

Associação Nacional de Pesquisa e Pós-Graduação em Ciência da Informação. 2014. Anais do ENANCIB. Paraiba: ANCIB. http://www. ancib.org.br/

Atkins, S. E. 1988. "Subject trends in library and information science research, 1975-1984”. Library Trends (36): 633-658. http://www. ideals.illinois.edu/ bitstream/handle/2142/7561/librarytrendsv36 i4c_opt.pdf? sequence $=1$

Braga, Gilda Maria. 1973. "Relações bibliométricas entre a frente de pesquisa (research front) e revisões da literatura". Ciência da Informação, Rio de Janeiro, 2 (1): 9-73. http://revista.ibict.br/ciinf/ article/view/20/20

- 1996. "A representação da informação na desconstrução do contexto". INFORMARE - Cadernos do Programa de Pós-Graduação em Ciência da Informação, Rio de Janeiro, 2 (2) (jul./dez.): 53-57. 
Braga, Katia. 2007. "Aspectos relevantes para a seleção de metodologia adequada à pesquisa social em Ciência da Informação", em Suzanna P. M. Mueller, Métodos para pesquisa em Ciência da Informação. Brasília: Thesaurus, 17-38.

Cano, V. 1999. "Bibliometric Overview of Library and Information Science Research in Spain". Journal of the American Society for Information Science 50 (8): 675-680. http://onlinelibrary.wiley.com/ doi/10.1002/(SICI)1097-4571(1999)50:8\%3C675::AID-ASI5\%3E 3.0.CO;2-B/pdf

Cordeiro, Helena C. D. 2012. Direito à informação: um estudo informétrico na base RVBI (1988/2010). Dissertação (Mestrado em Ciência da Informação) - PPGCI, UFF, Riode Janeiro.

Fonseca, E. N. (org.). 1986. Bibliometria: teoria e pratica. São Paulo: Cultrix: Ed da USP.

Hawkins, Donald T., Signe E. Larson e Bari Q. Caton. 2003. "Information science abstracts: tracking the literature of information science. Part 2 : a new taxonomy for information science". Journal of the American Society for Information Science and Technology 54 (8): 771-781.

Hjørland, B. 1998. "Theory and metatheory of Information Science: a new interpretation”. Journal of Documentation 54 (5) (dez.): 606621.

IBICT. 2011. Monitoramento internacional da produção científica em ciência da informação. Brasília, DF: IBICT, 2v. http://www.ibict.br/ publicacoes-e-institucionais/monitoramento-em-ci/Monitoramento_int_producao_cientifica_em_ci_v1.pdf e http://www. ibict.br/publicacoes-e-institucionais/monitoramento-em-ci/Monitoramento_int_producao_cientifica_em_ci_v2.pdf

Jarvelin, K. e P. Vakkari. 1993. "The evolution of library and information science 1965 - 1985: a content analysis of journal articles". Information Processing \& Management 29 (1): 129-144.

Lancaster, F. W. 2004. Indexação e resumos: teoria e prática. 2. ed. Brasília: Briquet de Lemos Livros.

Luiz, Alfredo J. B. 2002. "Meta-análise: definição, aplicações e sinergia com dados espaciais". Cadernos de Ciência E Tecnologia, Brasília, 19 (3) (set./dez.): 407-428.

Mukherjee, B. 2009. "Scholarly research in LIS open access electronic journals: A bibliometric study”. Scientometrics 80 (1): 169-196. https://www.bhu.ac.in /dlis/archive/169_10-mukh2055_1.pdf. DOI: 10.1007/s11192-008-2055-2

Preti, Oreste. 2006. "Tipos de pesquisa”, em $A$ aventura de ser estudante: um guia metodológico. 2. ed. rev., 7-14. Cuiabá: EdUMFT.

Salek, Lídia Martini Coelho Brandão. 2011. Artigos de periódicos e trabalhos apresentados em eventos: análise comparativa dos perfis temático-discursivos de diferentes fontes do campo informacional brasileiro. Trabalho de Conclusão de Curso (Graduação em Biblioteconomia)-Universidade Federal Fluminense, Niterói. 
Zins, Chaim. 2007. "Conceptions of Information Science". Journal of the American Society for Information Science and Technology 58 (3): $335-350$.

Para citar este artículo:

Amorim Neto, Miguel Romeu y Marcia Heloisa Tavares de Figueredo Lima. 2016. "O domínio de organização do conhecimento na base BRAPCI: uma análise estatística." Investigación Bibliotecológica: Archivonomía, Bibliotecología e Información 70: 83-104. http://dx. doi.org/10.1016/j.ibbai.2016.10.005

$\infty$ 